\title{
Late recurrences of pancreatic cancer in patients with long-term survival after pancreaticoduodenectomy
}

\author{
Andreas Minh Luu ${ }^{1 \#}$, Orlin Belyaev ${ }^{1 \#}$, Philipp Höhn ${ }^{1}$, Michael Praktiknjo ${ }^{2}$, Monika Janot ${ }^{1}$, \\ Waldemar Uhl ${ }^{1}$, Chris Braumann ${ }^{1}$ \\ ${ }^{1}$ Department of General and Visceral Surgery, St. Josef-Hospital, Ruhr - University Bochum, Gudrunstr. 56, 44791 Bochum, Germany; ${ }^{2}$ Department \\ of Internal Medicine, University of Bonn, Bonn, Germany \\ Contributions: (I) Conception and design: AM Luu, O Belyaev, C Braumann, W Uhl; (II) Administrative support: AM Luu, O Belyaev, C Braumann, \\ P Höhn, M Praktiknjo, M Janot; (III) Provision of study materials or patients: AM Luu, O Belyaev, P Höhn, M Praktiknjo, M Janot; (IV) Collection \\ and assembly of data: AM Luu; (V) Data analysis and interpretation: AM Luu, O Belyaev, C Braumann, M Praktiknjo, W Uhl; (VI) Manuscript \\ writing: All authors; (VII) Final approval of manuscript: All authors. \\ \#These authors contributed equally to this work. \\ Correspondence to: Andreas Minh Luu, MD. St. Josef Hospital, Ruhr-University Bochum, Gudrunstrasse 56, 44791 Bochum, Germany. \\ Email: a.luu@klinikum-bochum.de.
}

Background: Pancreatic cancer remains a relevant clinical problem due to poor prognosis. Even after curative pancreaticoduodenectomy tumor recurrences occur in up to $80 \%$. Risk factors for postoperative tumor recurrences have been identified before, but data on risk factors for tumor recurrences in patients with long-term-survival is scarce.

Methods: In this retrospective study consecutive long-term survival-patients (defined as at least 60 months survival) undergoing pancreaticoduodenectomy for pancreatic cancer from 2007-2014 were identified in the 2nd largest pancreatic surgery center in Germany. Clinical, pathohistological and laboratory values were analyzed to identify risk factors for tumor recurrence.

Results: Thirty-four of one-hundred-sixty-seven patients were identified as long-term-survival-patients in the study period. Of those, 10 patients (29.4\%) suffered from tumor recurrence. Lymph vessel invasion was identified as an independent risk factor $(\mathrm{P}=0.031$, hazard ratio $13.127,95 \%$ confidence interval: 1.270-135.698). Median postoperative time to tumor recurrence in long-term-survival-patients was 49 months. Overall survival after diagnosis of tumor recurrence was 33 months. $80 \%(\mathrm{~N}=8)$ of the patients were asymptomatic. Half of the patients $(\mathrm{N}=5)$ suffered from local disease, with $40 \%$ undergoing curative tumor resection. CA 19-9 levels were significantly elevated at $57 \mathrm{U} / \mathrm{mL}$ (normal $<27 \mathrm{U} / \mathrm{mL}$ ).

Conclusions: Tumor recurrence in long-term-survival-patients is typically asymptomatic. Especially longterm-survival-patients with lymph vessel invasion are more likely to develop tumor recurrence. Therefore, a structured follow-up program including CT-scans and CA 19-9 surveillance must be continued in all patients undergoing pancreaticoduodenectomy even in cases of long-term-survival.

Keywords: Pancreatic cancer (PC); pancreatic ductal adenocarcinoma; pancreaticoduodenectomy; recurrence; long-term survival

Submitted Oct 09, 2020. Accepted for publication Jan 17, 2021.

doi: 10.21037/jgo-20-433

View this article at: http://dx.doi.org/10.21037/jgo-20-433 


\section{Introduction}

Pancreatic cancer (PC) remains a devastating diagnosis for patients due to a poor prognosis. Unfortunately, PC is often diagnosed too late due to late development of symptoms. Surgical resection-the most important treatment modality for cure-is in general feasible in only less than $20 \%$ of patients at time of diagnosis (1-4). Despite technical, surgical, and oncological advances, pancreatic cancer is still ranked as the $4^{\text {th }}$ leading cancer related death and will soon be ranked $2^{\text {nd }}(5)$. Tumor related characteristics such as a large tumor, a high tumor grade, lymph-node metastases, a high level of carbohydrate antigen 19-9 (CA 19-9) and a cancer cell positive resection margin have been identified as negative prognostic factors for the overall postoperative survival (4).

International consensus guidelines recommend adjuvant chemotherapy following curative resection in most cases (6-8). Recent developments in oncologic treatment have shown significant improvement in overall postoperative survival of $28-54$ months $(9,10)$. Postoperative long-term survival (LTS) in PC is typically defined as a minimum survival of 60 months and is achieved in approximately $20 \%$ of patients after curative treatment (6,11-13).

Thus, up to $80 \%$ of all patients with PC suffer from tumor recurrence (TR) after resection with fatal outcome (14-16). They often present as local recurrences, or liver-, peritoneal-, or lung metastases with or without local recurrence $(4,17,18)$.

Despite an increasing amount of reports about improved overall survival following pancreaticoduodenectomy (PD), there is lacking information in literature about risk factors for TR in patients with LTS.

In this study we investigated the overall incidence and risk factors for tumor recurrence in $\mathrm{PC}$-patients with LTS following PD. We present the following article in accordance with the STROBE reporting checklist (available at: http://dx.doi.org/10.21037/jgo-20-433).

\section{Methods}

In this study, we retrospectively investigated data of all patients undergoing PD due to pancreatic ductal adenocarcinoma of the pancreatic head from January 2007 to December 2014 from the second largest pancreatic surgery center in Germany (St. Josef Hospital, RuhrUniversity Bochum). Patients diagnosed with other pancreatic cancer types like intraductal papillary mucinous neoplasia (IPMN) - associated carcinomas or acinar cell carcinomas were excluded. LTS was defined as a postoperative survival of at least 5 years (60 months). Primary endpoint was tumor recurrence of LTS patients. Secondary endpoint was overall survival of LTS-patients with TR. These patient data were compared to those of LTS-patients without TR (control group).

Follow-up to evaluate LTS in all patients was performed until August 2020. Patient data was gathered from our prospective database.

Discharge letters and all relevant documents were obtained from other institutions or from the patients' general practitioner if the treatment and follow-up was continued elsewhere. Last, all patients or their relatives underwent a phone-interview to evaluate the overall duration of postoperative survival.

\section{Surgical approach}

The standard surgical approach in resectable pancreatic head cancer is a pylorus-preserving pancreaticoduodenectomy. Further, the standard type of pancreatic anastomosis is a doublelayered end-to-side duct-to-mucosa pancreaticojejunostomy as described by Warren and Catell (19).

\section{Statistical analysis}

Data were expressed as percentages and the median with interquartile range. Comparison of LTS-patients with TR to those without TR was performed using a two-tailed chi-square test, a Fisher's exact test, or a Mann-Whitney $\mathrm{U}$ test, as appropriate. A binary logistic regression model was applied for multivariate analysis to identify risk factors for TR in LTS-patients. All variables being significant in univariate analysis were entered in the multivariate analysis. Statistical significance was present in the case of $\mathrm{P}$ values $<0.05$. Statistical analysis was performed with SPSS 21.0 (IBM Corp., Armonk, NY, USA).

\section{Ethical statement}

This study has been approved by the institutional review board of the Ruhr-University of Bochum (NO: 19-6771$\mathrm{BR})$. Because of the retrospective nature of the study, the requirement for informed consent was waived. The study was conducted in accordance with the Declaration of Helsinki (as revised in 2013). 
Table 1 Epidemiologic data of all long-term survival patients and all patients with or without tumor recurrences

\begin{tabular}{|c|c|c|c|c|}
\hline & All patients & Recurrence & No recurrence & $P$ value \\
\hline Survival of all LTS patients (months, median, IQR) & $102(80-142)$ & $79(65-92)$ & - & - \\
\hline Time of recurrence (months) & - & $49(30-68)$ & - & - \\
\hline Time of late recurrence (months) & - & $68(56-68)$ & - & - \\
\hline \multicolumn{5}{|l|}{ Age at time of primary surgery, median (\%) } \\
\hline$\leq 65$ & $18(53 \%)$ & - & - & - \\
\hline$>65$ & $16(47 \%)$ & - & - & - \\
\hline \multicolumn{5}{|l|}{ Sex } \\
\hline \multicolumn{5}{|l|}{ ASA score } \\
\hline I & $5(14.7 \%)$ & $2(20 \%)$ & $3(12.5 \%)$ & 0.376 \\
\hline II & $15(44.1 \%)$ & $5(50 \%)$ & $10(41.7 \%)$ & \\
\hline III & $14(41.2 \%)$ & $3(30 \%)$ & $11(45.8 \%)$ & \\
\hline \multicolumn{5}{|l|}{ IV } \\
\hline \multicolumn{5}{|l|}{ Additional potential risk factors } \\
\hline Diabetes mellitus & $12(35.3 \%)$ & $3(30 \%)$ & $9(38 \%)$ & 0.681 \\
\hline Cardiovascular diseases & $16(47.1 \%)$ & $2(20 \%)$ & $14(58 \%)$ & 0.044 \\
\hline Preoperative stenting of the CBD & $25(73.5 \%)$ & $7(70 \%)$ & $18(75 \%)$ & 0.767 \\
\hline \multicolumn{5}{|l|}{ Preoperative laboratory values } \\
\hline Initial CA 19-9 U/mL, median (IQR) & $73.0(20.0-163)$ & $88.5(20-182)$ & $73(19-173)$ & 0.880 \\
\hline CA $19-9$ at time of recurrence & - & $57(17-176)$ & - & - \\
\hline
\end{tabular}

LTS, long-term survival; IQR, interquartile range; ASA, American Society of Anesthesiologists; CBD, common bile duct; CA 19-9, carbohydrate antigen 19-9.

\section{Results}

Thirty-four out of 167 patients were identified as LTSpatients following PD (20.4\%) in the study period. Of those 10 patients (29.4\%) suffered from TR (Table 1). Median time to TR was 49 months (IQR: 30-68, Figure 1), whereas median time to late-recurrence, i.e., TR after postoperative month 60, was 68 months. Overall survival after detection of recurrent disease was 33 months [15-43]. Male to female ratio was $0.43(\mathrm{P}=0.681)$. No significant differences in LTSpatients with or without TR in terms of comorbidities were observed $(\mathrm{P}=0.376)$ except for cardiovascular diseases, being more common patients without TR $(\mathrm{P}=0.044)$. CA 19-9 levels were significantly elevated at time of TR (median level: $57 \mathrm{U} / \mathrm{mL}$, normal $<27 \mathrm{U} / \mathrm{mL}$ ). 


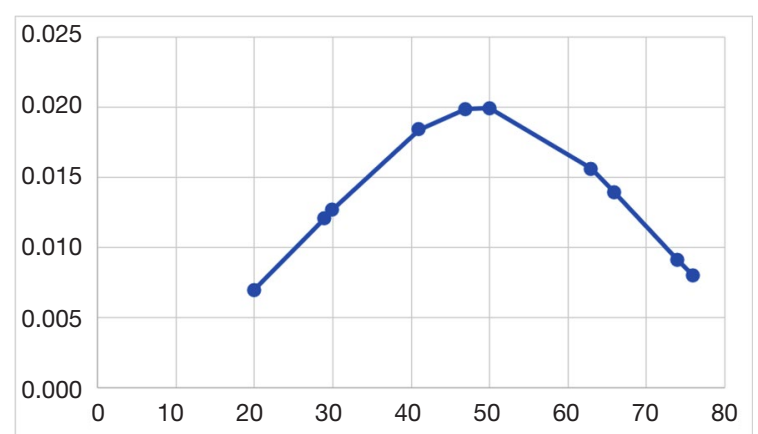

Figure 1 Graph demonstrating the normal distribution of tumor recurrences of patients who underwent pancreaticoduodenectomy due to pancreatic cancer. Y-axis: normal distribution function. $\mathrm{X}$-axis: postoperative months following pancreaticoduodenectomy.

\section{Location of tumor recurrence and treatment}

Tumor recurrence in LTS-patients was mainly diagnosed in routine follow-up studies without symptoms $(80 \%, \mathrm{~N}=8)$. Only 2 patients $(20 \%)$ presented with symptoms due to small bowel obstruction or TR at the pancreaticojejunostomy.

Half of the patients suffered from local TR $(50 \%$, Table 2). The other half suffered from liver- $(\mathrm{N}=4)$, lung$(\mathrm{N}=2)$ or peritoneal $(\mathrm{N}=2)$ metastases. Surgical treatment was performed in $60 \%$. Forty percent underwent curative surgery in terms of a completion pancreatectomy $(20 \%)$, a Re-Do-pancreaticoduodenectomy (10\%) or a liver segment resection $(10 \%)$. Palliative surgery was performed twice (20\%) due to small bowel obstruction.

Chemotherapy was either adjuvant $(40 \%)$ and gemcitabine- or fluorouracil-based or palliative (40\%) and FOLFIRINOX-[folinic acid ([leucovorin], fluorouracil, irinotecan, and oxaliplatin) or gemcitabine-based. Twenty percent received no chemotherapy.

\section{Histopathological features}

Patients with lymph vessel invasion suffered significantly more often from TR compared to other patients $(\mathrm{P}=0.019$, Table 3). Furthermore, those patients had higher rates of venous and perineural invasion. However, statistically significant differences were not present. All patients with TR were classified T3 compared to $83.3 \%$ in the control group $(\mathrm{P}=0.176)$, in which 4 patients were classified $\mathrm{T} 1$ or T2. R0 resection was achieved in $80 \%(\mathrm{~N}=8)$, whereas $20 \%$ $(\mathrm{N}=2)$ were classified R1. No differences in terms of AJCC-
Table 2 Location of tumor recurrence and treatment

\begin{tabular}{|c|c|}
\hline & TR patients [\%] \\
\hline \multicolumn{2}{|l|}{ Location of tumor recurrence } \\
\hline Local tumor recurrence & $5[50]$ \\
\hline Local recurrence + anywhere & $3[30]$ \\
\hline Metastases, no local recurrence & $5[50]$ \\
\hline Liver & $4[40]$ \\
\hline Peritoneum & $2[20]$ \\
\hline Lung & $4[40]$ \\
\hline Surgical treatment & $6[60]$ \\
\hline Curative intention & $4[40]$ \\
\hline Completion pancreatectomy & $2[20]$ \\
\hline Atypical liver resection & $1[10]$ \\
\hline Re-Do pancreaticoduodenectomy & $1[10]$ \\
\hline Palliative surgery & $2[20]$ \\
\hline \multicolumn{2}{|l|}{ Chemotherapy } \\
\hline Adjuvant & $4[40]$ \\
\hline Gemcitabine & $1[10]$ \\
\hline Gemcitabine + oxaliplatin & $1[10]$ \\
\hline 5 Fluorouracil & $1[10]$ \\
\hline 5 Fluorouracil, oxaliplatin & $1[10]$ \\
\hline Palliative & $4[40]$ \\
\hline FOLFIRINOX & $2[20]$ \\
\hline Gemcitabine & $1[10]$ \\
\hline Gemcitabine + orlitinib & $1[10]$ \\
\hline No chemotherapy & $2[20]$ \\
\hline
\end{tabular}

TR, tumor recurrence.

stage (American Joint Committee on Cancer), resection margin or grading were observed.

\section{Tumor re-recurrence}

Two out of ten patients (20\%) with TR suffered from rerecurrence following curative surgery (Table 4). Median interval between surgery and re-recurrence was 33 months. Overall median survival was 83.5 months (Figure 2). One patient underwent palliative chemotherapy with oxaliplatin and fluorouracil. The other one received best supportive care only. Median CA $19-9$ was $285 \mathrm{U} / \mathrm{mL}$. 
Table 3 Histopathological features

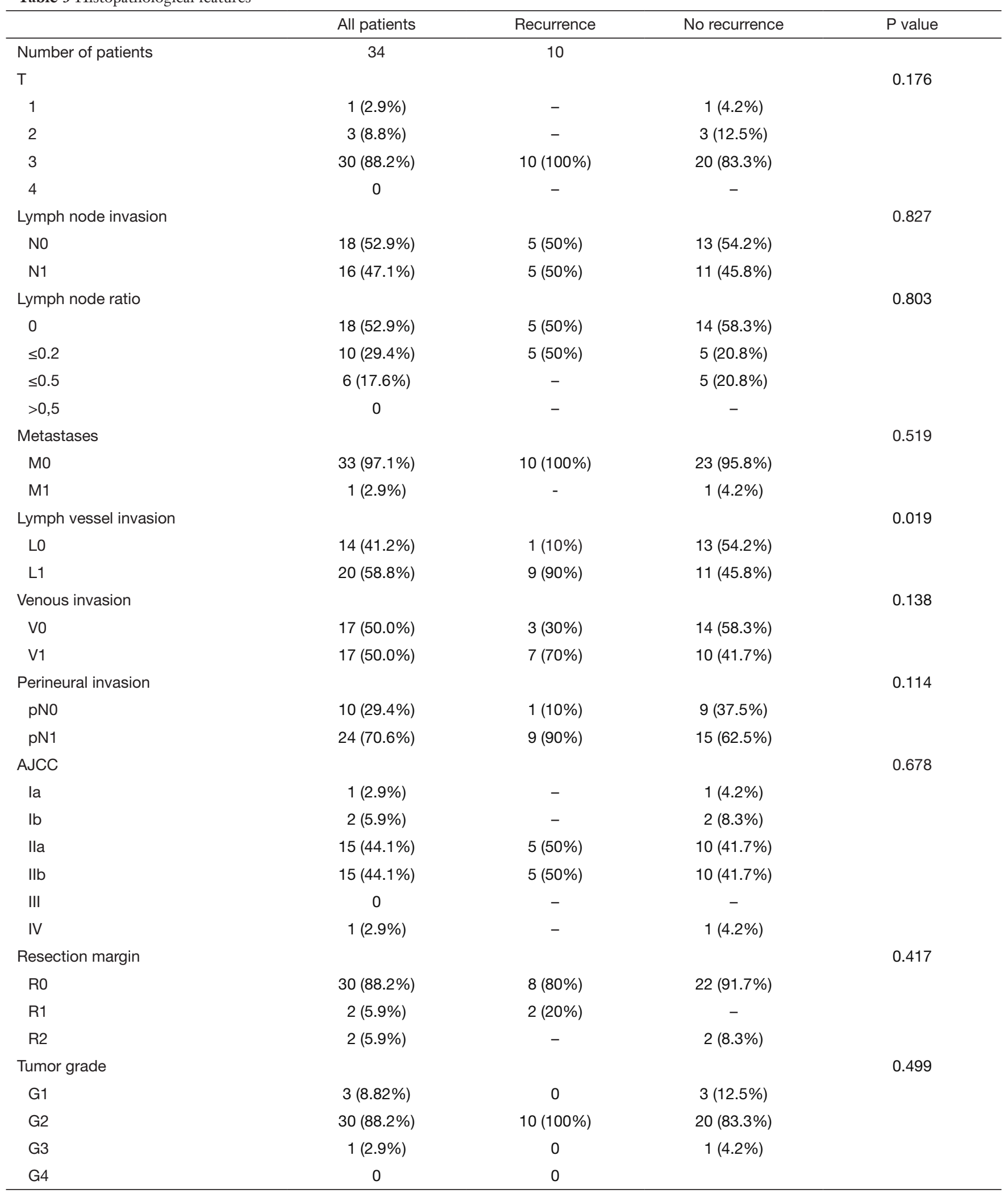

AJCC, American Joint Committee on Cancer. 
Table 4 Data of patients with tumor re-recurrences

\begin{tabular}{lc}
\hline & TRR \\
\hline Re-recurrences, N & 2 \\
$\begin{array}{l}\text { Postoperative time of TR from after } \\
\text { primary surgery }\end{array}$ & $49(30-68)$ \\
Time of TRR after TR & $33(15-33)$ \\
Survival, (months) & $83.5(79-83.5)$ \\
Surgery & $0(0 \%)$ \\
CTx (N=1) & Oxaliplatin + 5 \\
CA 19-9 & Fluorouracil \\
CA 19-9 with TRR & $546(42-546)$ \\
\hline
\end{tabular}

TR, tumor recurrence; TRR, tumor re-recurrence; CTx, chemotherapy; CA, carbohydrate antigen.

Table 5 Binary logistic regression analysis for multivariate analysis

\begin{tabular}{lccc}
\hline & Hazard ratio & $\begin{array}{c}95 \% \text { confidence } \\
\text { interval }\end{array}$ & P value \\
\hline $\begin{array}{l}\text { Lymph vessel } \\
\text { invasion }\end{array}$ & 13.127 & $1.270-135.698$ & 0.031 \\
$\begin{array}{l}\text { Cardiovascular } \\
\text { diseases }\end{array}$ & 0.141 & $0.021-0.963$ & 0.046 \\
\hline
\end{tabular}

\section{Multivariate analysis}

Binary logistic regression analysis was performed for multivariate analysis (Table 5). Lymph vessel invasion and cardiovascular diseases were significant in univariate analysis and thus entered in the multivariate model. Lymph vessel invasion was identified as an independent risk factor for TR in patients with LTS $(\mathrm{P}=0.031$, hazard ratio $13.127,95 \%$ confidence interval: 1.270-135.698). Cardiovascular diseases were also identified as influencing factors $(\mathrm{P}=0.046$, hazard ratio $0.141,95 \%$ confidence interval: $0.021-0.963$ ).

\section{Discussion}

This study demonstrates that PC remains a serious disease which patients and physicians should always stay aware of even in cases of LTS.

Unfortunately, up to $80 \%$ of the patients with PC suffer from TR which often leads to patient demise within 2 years of curative resection $(15,16,18,20,21)$. Our data demonstrate that even LTS patients are not spared from TR which occurs

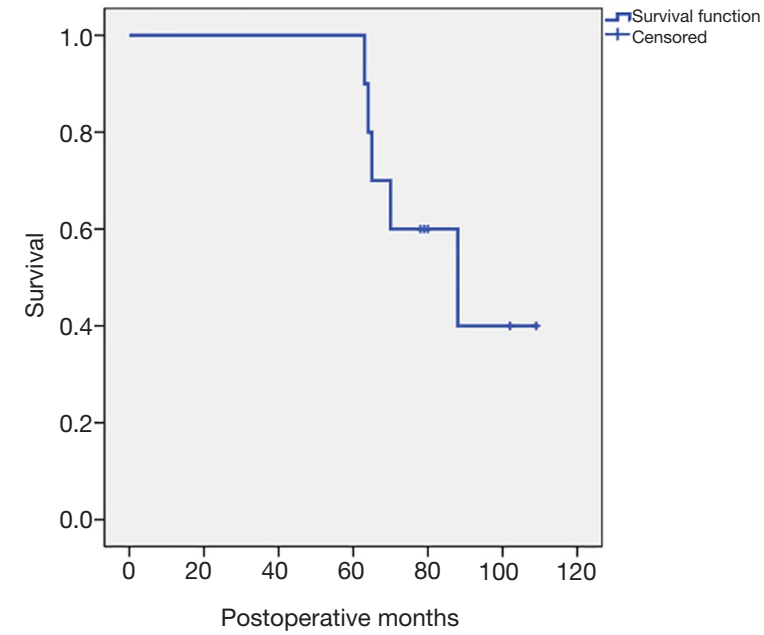

Figure 2 Kaplan-Meier curve demonstrating overall survival of long-term survival patients with tumor recurrence.

in approximately $30 \%$. Undetected micrometastases are typically too small for detection with radiographic imaging and can evolve undetected over time (22). Therefore, even pathologic complete response following neoadjuvant treatment in patients with $\mathrm{PD}$ can unfortunately be followed by TR (1,23-25). Thus, pancreatic cancer must be considered a systemic disease even at a stage of local resectability (18). According to international guideline recommendations the combination of curative resection and additional chemotherapy treatment is crucial $(4,18)$. Median disease-free survival is $12-13$ months and can nowadays be increased up to 21.6 months with modified FOLFIRINOX treatment $(10,18)$.

Most LTS-patients (80\%) tend to be asymptomatic at the time of TR compared to non-LTS patients. The latter group tends to suffer in $76 \%$ from symptoms compared to $20 \%$ in LTS-patients (15). This can be explained by the more aggressive growth pattern of PC in non-LTS patients with an increased amount of lymph node-, venous- or perineural invasion as well as higher tumor gradings (4). Treatment rates in case of recurrent cancer are unequally reported in literature. Daamen et al. report a 35\% treatment rate compared to a best supportive care rate of $65 \%$ : main causes were the patient's wish $(40 \%)$ and poor overall performance status (30\%) (15). However, another study by Gbolahan et al. investigating data of 435 patients showed a $63 \%$ chemotherapy rate and a $32 \%$ best supportive care rate (26).

Relevant risk factors for local TR are a negative resection 
margin as well as existence and extension of lymphogenous metastasis $(20,22,27,28)$. Further relevant factors affecting the overall survival were perioperative treatment by chemotherapy or radiotherapy (26).

In our study, we identified lymph vessel invasion as an independent risk factor for TR in patients with LTS. Presence of cardiovascular diseases was associated with longer survival. However, we believe this effect occurred only by chance due to the low case number as there is no indication in literature for this effect.

Isolated TR allowing surgical resection was present in $40 \%$ in our cohort and higher than in literature (20-30\%) $(18,22)$. Resection of local TR is technically demanding due to adhesions, local tumor mass and modified anatomical surrounding. In those cases, surgery should be performed in high-volume pancreatic surgery centers which then is associated with a lower grade of morbidity (18-29\%). Treatment options comprise completion pancreatectomy, Re-Do-pancreaticoduodenectomy or liver segment resection and can finally lead to a significant improvement of overall survival compared to single chemotherapy or radiotherapy (26 vs. 14 months) (21,29,30). Isolated resection of pancreatic TR leads to median overall survival of 16-32 months (21,30-32). Importantly, according to international guidelines, resection of recurrent disease is not considered a standard-approach. Other local treatment options being discussed in literature are radiotherapy and irreversible electroporation in selected patients $(20,33)$.

Commonly, surgical resection cannot be performed in patients with TR, because half of the patients already suffer from multilocular disease or distant metastases, which are present in up to $62 \%(15,28)$. As a meta-analysis by Tanaka et al. investigating data of 17,313 patients demonstrated, liver metastases occur in $26.5 \%$, lung metastases in $11.4 \%$ and peritoneal metastases in $13.5 \%$ following curative resection (22). Typically, liver and lung metastases are associated with a shorter recurrence free survival as well as a shorter overall survival. Daamen et al. described in a large nationwide Dutch study with 836 patients a significant increase of overall survival in patients with tumor recurrence who received additional treatment underlining the results of Tzeng, Tjaden and Nordby (15,34-36). Furthermore, those studies demonstrated an increase of life quality compared to patients receiving best supportive care only. Typical chemotherapy regimens are FOLFIRINOX (68\%) followed by gemcitabine \pm nab-paclitaxel (15).

Our data present significant higher post-recurrence survival rates compared to current survival data in the literature. Median survival period after detection of TR was 33 months compared to a median of 7 months reported in the literature due to delayed diagnosis of TR (26). In cases of a low overall performance status, patients receive best supportive care only. Median survival has been reported to be 3 months, comparable to patients being primary diagnosed with an advanced pancreatic cancer with either brain or umbilical metastases $(37,38)$. These data underline the aggressive growth pattern and common multifocal recurrences of $\mathrm{PC}$.

One important requirement for additional treatment is a good ECOG performance status (39). Therefore, a timely detection of tumor recurrence is crucial for patient survival. As our results demonstrate, most tumor recurrences in LTS patients are asymptomatic (80\%). Only $20 \%$ of the patients were complaining of abdominal pain. Currently, the German guideline on pancreatic cancer does not recommend regular postoperative follow up investigations (7). However, patients with structured follow up survive 25 months compared to 15 months in patients who received symptomatic follow up only (15). Additionally, to the study group of Daamen, an increasing number of clinicians suggest a structured postoperative surveillance $(22,34,35)$. Focusing on clinical symptoms without routine imaging commonly leads to diagnosis of TR at an advanced stage with a decreased chance for successful secondary treatment (40). A cost-effectiveness study by Tzeng et al. demonstrated that a surveillance every 6 months is the most cost-effective strategy (41).

As most TR in our LTS-cohort occurred relatively late at the $49^{\text {th }}$ postoperative month, we strongly recommend a continuous follow-up program for all patients undergoing curative resection for $\mathrm{PC}$ to detect TR in time for surgical and/or chemotherapeutical treatment. Late recurrences appearing after 5 years of uneventful courses exist as well as re-recurrences following curative tumor resection of a recurrent tumor. This emphasizes the hazardous nature of pancreatic cancer. Most importantly, even in patients with LTS, the surveillance should be continued after the fifth year. One important problem in radiographic findings-especially in asymptomatic patients-is that TR cannot always be differentiated from unspecific postoperative tissue alterations. Our structured follow-up program consists of abdominal CT-scans every 6 months, abdominal ultrasounds every 3 months and testing of CA 19-9 levels every 3 months for the first five postoperative years. As Azizian et al. demonstrated, our data confirm that CA 19-9 is the most important laboratory value indicating 
TR and must be monitored regularly (42). If no tumor recurs within five years, the radiographic control interval can be prolonged to every 12 months but should never be discontinued. The CA 19-9 surveillance should be continued every 6 months.

This study is limited by the small cohort of LTSpatients with TR, because LTS by itself is relatively rare. Furthermore, no standardized adjuvant treatment was given.

Future studies should enroll a larger number of patients to identify further risk factors for tumor recurrence. Furthermore, analysis of the impact and best chemotherapy regimen in patients with TR is required. Decrease of tumor load as well as the lowest level of medical side-effects are the main goals.

In conclusion, our data shows that tumor recurrence in LTS-patients is common (especially in patients with lymph vessel invasion) and typically asymptomatic. Therefore, we suggest a structured follow up of CA 19-9, abdominal ultrasound, and CT-scans even after the fifth postoperative year.

\section{Acknowledgments}

Funding: None.

\section{Footnote}

Reporting Checklist: The authors have completed the STROBE reporting checklist. Available at: http://dx.doi. org/10.21037/jgo-20-433

Data Sharing Statement: Available at: http://dx.doi. org/10.21037/jgo-20-433

Conflicts of Interest: All authors have completed the ICMJE uniform disclosure form (available at: http://dx.doi. org/10.21037/jgo-20-433). The authors have no conflicts of interest to declare.

Ethical Statement: The authors are accountable for all aspects of the work in ensuring that questions related to the accuracy or integrity of any part of the work are appropriately investigated and resolved. This study has been approved by the institutional review board of the Ruhr-University of Bochum (No: 19-6771-BR). Because of the retrospective nature of the study, the requirement for informed consent was waived. The study was conducted in accordance with the Declaration of
Helsinki (as revised in 2013).

Open Access Statement: This is an Open Access article distributed in accordance with the Creative Commons Attribution-NonCommercial-NoDerivs 4.0 International License (CC BY-NC-ND 4.0), which permits the noncommercial replication and distribution of the article with the strict proviso that no changes or edits are made and the original work is properly cited (including links to both the formal publication through the relevant DOI and the license). See: https://creativecommons.org/licenses/by-nc-nd/4.0/.

\section{References}

1. Luu AM, Hoehn P, Vogel SR, et al. Pathologic Complete Response of Pancreatic Cancer following Neoadjuvant FOLFIRINOX Treatment in Hepatic Metastasized Pancreatic Cancer. Visc Med 2019;35:387-91.

2. Chari ST, Kelly K, Hollingsworth MA, et al. Early detection of sporadic pancreatic cancer: summative review. Pancreas 2015;44:693-712.

3. Wagner M, Redaelli C, Lietz M, et al. Curative resection is the single most important factor determining outcome in patients with pancreatic adenocarcinoma. Br J Surg 2004;91:586-94.

4. Hidalgo M. Pancreatic cancer. N Engl J Med 2010;362:1605-17.

5. Siegel RL, Miller KD, Jemal A. Cancer statistics, 2020. CA Cancer J Clin 2020;70:7-30.

6. Oettle H, Neuhaus P, Hochhaus A, et al. Adjuvant chemotherapy with gemcitabine and long-term outcomes among patients with resected pancreatic cancer: the CONKO-001 randomized trial. JAMA 2013;310:1473-81.

7. Seufferlein T, Porzner M, Becker T, et al. [S3guideline exocrine pancreatic cancer]. Z Gastroenterol 2013;51:1395-440.

8. Tempero MA, Malafa MP, Al-Hawary M, et al. Pancreatic Adenocarcinoma, Version 2.2017, NCCN Clinical Practice Guidelines in Oncology. J Natl Compr Canc Netw 2017;15:1028-61.

9. Neoptolemos JP, Palmer DH, Ghaneh P, et al. Comparison of adjuvant gemcitabine and capecitabine with gemcitabine monotherapy in patients with resected pancreatic cancer (ESPAC-4): a multicentre, open-label, randomised, phase 3 trial. Lancet 2017;389:1011-24.

10. Conroy T, Hammel P, Hebbar M, et al. FOLFIRINOX or Gemcitabine as Adjuvant Therapy for Pancreatic Cancer. N Engl J Med 2018;379:2395-406. 
11. Kardosh A, Lichtensztajn DY, Gubens MA, et al. LongTerm Survivors of Pancreatic Cancer: A California Population-Based Study. Pancreas 2018;47:958-66.

12. Stark AP, Sacks GD, Rochefort MM, et al. Long-term survival in patients with pancreatic ductal adenocarcinoma. Surgery 2016;159:1520-7.

13. Katz MH, Wang H, Fleming JB, et al. Long-term survival after multidisciplinary management of resected pancreatic adenocarcinoma. Ann Surg Oncol 2009;16:836-47.

14. Bednar F, Pasca di Magliano M. Chemotherapy and Tumor Evolution Shape Pancreatic Cancer Recurrence after Resection. Cancer Discov 2020;10:762-4.

15. Daamen LA, Groot VP, Besselink MG, et al. Detection, Treatment, and Survival of Pancreatic Cancer Recurrence in the Netherlands: A Nationwide Analysis. Ann Surg 2020. [Epub aead of print]. doi: 10.1097/ SLA.0000000000004093.

16. D'Haese JG, Renz BW, Ilmer M, et al. Surgery for isolated local recurrence and metachronous oligometastasis in pancreatic cancer. Chirurg 2020;91:628-35.

17. Griffin JF, Smalley SR, Jewell W, et al. Patterns of failure after curative resection of pancreatic carcinoma. Cancer 1990;66:56-61.

18. Strobel O, Buchler MW. Surgery of pancreatic cancer: techniques to avoid local recurrence. Chirurg 2020;91:615-27.

19. Warren KW, Cattell RB. Basic techniques in pancreatic surgery. Surg Clin North Am 1956;36:707-24.

20. Groot VP, Rezaee N, Wu W, et al. Patterns, Timing, and Predictors of Recurrence Following Pancreatectomy for Pancreatic Ductal Adenocarcinoma. Ann Surg 2018;267:936-45.

21. Yamada S, Kobayashi A, Nakamori S, et al. Resection for recurrent pancreatic cancer in the remnant pancreas after pancreatectomy is clinically promising: Results of a project study for pancreatic surgery by the Japanese Society of Hepato-Biliary-Pancreatic Surgery. Surgery 2018;164:1049-56.

22. Tanaka M, Mihaljevic AL, Probst P, et al. Meta-analysis of recurrence pattern after resection for pancreatic cancer. $\mathrm{Br}$ J Surg 2019;106:1590-601.

23. Luu AM, Herzog T, Hoehn P, et al. FOLFIRINOX treatment leading to pathologic complete response of a locally advanced pancreatic cancer. J Gastrointest Oncol 2018;9:E9-E12.

24. Blair AB, Yin LD, Pu N, et al. Recurrence in Patients Achieving Pathological Complete Response After Neoadjuvant Treatment for Advanced Pancreatic Cancer.
Ann Surg 2019. [Epub aead of print]. doi: 10.1097/ SLA.0000000000003570.

25. Höhn P BC, Nöpel-Dünnebacke S, Munding J, et al. Recurrence of Pancreatic Ductal Adenocarcinoma after Complete Histopathological Remission Caused by FOLFIRINOX. Visc Med 2020. doi: 10.1159/000509231

26. Gbolahan OB, Tong Y, Sehdev A, et al. Overall survival of patients with recurrent pancreatic cancer treated with systemic therapy: a retrospective study. BMC Cancer 2019;19:468.

27. Jones RP, Psarelli EE, Jackson R, et al. Patterns of Recurrence After Resection of Pancreatic Ductal Adenocarcinoma: A Secondary Analysis of the ESPAC-4 Randomized Adjuvant Chemotherapy Trial. JAMA Surg 2019;154:1038-48.

28. Honselmann KC, Pergolini I, Castillo CF, et al. Timing But Not Patterns of Recurrence Is Different Between Node-negative and Node-positive Resected Pancreatic Cancer. Ann Surg 2020;272:357-65.

29. Luu AM, Krasemann L, Fahlbusch T, et al. Facing the surgeon's nightmare: Incidence and Management of postoperative pancreatic fistulas grade $\mathrm{C}$ after pancreaticoduodenectomy based on the updated definition of the International Study Group (ISGPS). J Hepatobiliary Pancreat Sci 2020;27:171-81.

30. Groot VP, van Santvoort HC, Rombouts SJ, et al. Systematic review on the treatment of isolated local recurrence of pancreatic cancer after surgery; reresection, chemoradiotherapy and SBRT. HPB (Oxford) 2017;19:83-92.

31. Strobel O, Hartwig W, Hackert T, et al. Re-resection for isolated local recurrence of pancreatic cancer is feasible, safe, and associated with encouraging survival. Ann Surg Oncol 2013;20:964-72.

32. Kleeff J, Reiser C, Hinz U, et al. Surgery for recurrent pancreatic ductal adenocarcinoma. Ann Surg 2007;245:566-72.

33. Sperti C, Moletta L, Merigliano S. Multimodality treatment of recurrent pancreatic cancer: Mith or reality? World J Gastrointest Oncol 2015;7:375-82.

34. Tjaden C, Michalski CW, Strobel O, et al. Clinical Impact of Structured Follow-up After Pancreatic Surgery. Pancreas 2016;45:895-9.

35. Nordby T, Hugenschmidt H, Fagerland MW, et al. Follow-up after curative surgery for pancreatic ductal adenocarcinoma: asymptomatic recurrence is associated with improved survival. Eur J Surg Oncol 2013;39:559-66.

36. Tzeng CW, Fleming JB, Lee JE, et al. Yield of clinical 
and radiographic surveillance in patients with resected pancreatic adenocarcinoma following multimodal therapy. HPB (Oxford) 2012;14:365-72.

37. Luu AM, Künzli B, Hoehn P, et al. Prognostic value and impact of cerebral metastases in pancreatic cancer. Acta Chir Belg 2020;120:30-4.

38. Luu AM, Meurer K, Herzog T, et al. Surprising Twist in the Plot - Sister Mary Joseph's Nodule of Pancreatic Cancer Mimicking Wound Infection after Umbilical Hernia Repair. J Gastrointest Cancer 2019;50:113-5.

39. Tas F, Sen F, Odabas H, et al. Performance status of patients is the major prognostic factor at all stages of

Cite this article as: Luu AM, Belyaev O, Höhn P, Praktiknjo M, Janot M, Uhl W, Braumann C. Late recurrences of pancreatic cancer in patients with long-term survival after pancreaticoduodenectomy. J Gastrointest Oncol 2021;12(2):474483. doi: 10.21037/jgo-20-433 pancreatic cancer. Int J Clin Oncol 2013;18:839-46.

40. Groot VP, Daamen LA, Hagendoorn J, et al. Use of imaging during symptomatic follow-up after resection of pancreatic ductal adenocarcinoma. J Surg Res 2018;221:152-60.

41. Tzeng CW, Abbott DE, Cantor SB, et al. Frequency and intensity of postoperative surveillance after curative treatment of pancreatic cancer: a cost-effectiveness analysis. Ann Surg Oncol 2013;20:2197-203.

42. Azizian A, Ruhlmann F, Krause T, et al. CA19-9 for detecting recurrence of pancreatic cancer. Sci Rep 2020;10:1332. 